Sharif University of Technology
Scientia Iranica
Transactions E: Industrial Engineering
whw.scientiairanica.com
IRAN I C A

Research Note

\title{
A simple solution procedure for solving the multi-delivery policy into economic production lot size problem with partial rework
}

\author{
K.-J. Chung ${ }^{\mathrm{a}, \mathrm{b}, \mathrm{c}}$, P.-Sh. Ting ${ }^{\mathrm{c}}$ and L.E. Cárdenas-Barrón ${ }^{\mathrm{d}, *}$ \\ a. College of Business, Chung Yuan Christian University, Chung Li, Taiwan, R.O.C. \\ b. National Taiwan University of Science and Technology, Taipei, Taiwan, R.O.C. \\ c. Department of International Business Management, Shih Chien University, Taipei, Taiwan, R.O.C. \\ d. School of Engineering and Sciences, Tecnológico de Monterrey, Ave. Eugenio Garza Sada 2501 Sur, C.P. 64849, Monterrey, \\ Nuevo León, México.
}

Received 16 January 2015; received in revised form 12 July 2016; accepted 24 September 2016

\section{KEYWORDS}

Replenishment lot

size;

Multiple shipments;

Manufacturing;

Rework;

Scrap;

Inventory.

\begin{abstract}
Recently, an alternative multi-delivery policy into imperfect Economic Production Quantity (EPQ) inventory model with partial rework has been proposed, which considers the number of shipments as a fixed and given value. This paper, treating the longrun average costs per unit time as a function of replenishment lot size $Q$ and the number of shipments $n$, adopts the differential calculus approach to get the optimal solution of $Q$ and $n$ jointly. In numerical examples, it is illustrated that the solution procedure is simple and accurate.

(C) 2017 Sharif University of Technology. All rights reserved.
\end{abstract}

\section{Introduction}

An interesting research topic in inventory theory is the development of inventory models when the manufacturing system produces defective items which can be reworked. Within this context, Chiu et al. [1] proposed a multi-delivery policy into an imperfect Economic Production Quantity (EPQ) inventory model with partial rework. Essentially, they treat their long-run average cost as a function of the replenishment lot size, $Q$. Consequently, the long-run average cost function of Chiu et al. [1] is a function of only one decision variable, $Q$. Furthermore, they consider the number

\footnotetext{
*. Corresponding author. Tel.: +52 8183284235 ; Fax: +528183284153

E-mail address: lecarden@itesm.mx (L.E. Cárdenas-Barrón)
}

of shipments, $n$, as a constant. Conversely, CárdenasBarrón et al. [2] generalized the model of Chiu et al. [1] to allow the number of shipments, $n$, to be a decision variable, such that the long-run average cost is a function of two decision variables, $Q$ and $n$. A solution procedure to determine both $Q$ and $n$ is also proposed.

Following the approach of Cárdenas-Barrón et al. [2], this paper adopts the calculus approach to obtain the optimal solution of $Q$ and $n$ jointly. The solution procedure simplifies that of Cárdenas-Barrón et al. [2]. Research on EPQ inventory models with partial rework can be found in Cárdenas-Barrón et al. [2], Chiu et al. [1], Sana [3], Sana [4], Sana and Goyal [5], and their references.

In the following paper, Section 2 presents the mathematical formulation of the inventory model. Section 3 proposes two theorems to locate the optimal solution. Section 4 illustrates the use of the theorems 
with numerical examples. Finally, a remark conclusion is given.

\section{Model formulation}

We adopt all of the notation and assumptions described in Chiu et al. [1] and Cárdenas-Barrón et al. [2] to establish a new manufacturing model which allows the number of shipments $n$ to be a decision variable. Following Chiu et al. [1] and Cárdenas-Barrón et al. [2], we can get the long-run average costs, $E[n, Q]$, per unit time for the new manufacturing model as follows:

$$
E[n, Q]=\psi_{3}+\psi_{2}(n) Q^{-1}+\psi_{1}(n) Q,
$$

where:

$$
\begin{aligned}
& \psi_{3}=\frac{C \lambda}{1-\theta E[x]}+C_{T} \lambda+\frac{C_{S} E[x] \theta \lambda}{1-\theta E[x]} \\
& +\frac{C_{R} E[x](1-\theta) \lambda}{1-\theta E[x]} \\
& \psi_{2}(n)=\frac{\left(K+(n+1) K_{1}\right) \lambda}{1-\theta E[x]}, \\
& \psi_{1}(n)=\frac{h \lambda^{2}}{2 P(1-\theta E[x])}\left[\frac{2 \lambda}{P^{2}} E\left(\frac{1}{1-x}\right)-\frac{1}{P}\right. \\
& +\frac{(1-\theta)}{P_{1}}\left[\frac{4 \lambda}{P} E\left(\frac{x}{1-x}\right)\right. \\
& \left.\left.+\frac{2 \lambda(1-\theta)}{P_{1}} E\left(\frac{x^{2}}{1-x}\right)-2 E(x)\right]\right] \\
& +\frac{h}{2(1-\theta E[x])}\left\{-\frac{\lambda[E(x)]^{2}(1-\theta)^{2}}{P_{1}}\left[1+\frac{\lambda}{P_{1}}\right]\right. \\
& \left.+[1-\theta E(x)]^{2}-\frac{\lambda[1-2 \theta E(x)]}{P}\right\} \\
& -\frac{h}{2 n(1-\theta E(x))}\left\{[1-\theta E(x)]^{2}-\frac{\lambda}{P}\left[2-\frac{\lambda}{P}\right]\right. \\
& +\frac{\lambda E(x)(1-\theta)}{P_{1}}[-2[1-\theta E(x)] \\
& \left.+\frac{2 \lambda}{P}+\frac{\lambda E(x)(1-\theta)}{P_{1}}\right\} \\
& +\left\{\frac{1}{2(1-\theta E(x))}\right\} \frac{h_{1}(E[x])^{2} \lambda(1-\theta)^{2}}{P_{1}} \\
& =\frac{1}{2(1-\theta E[x])}\left\{A-\frac{B}{n}+D\right\} \text {, }
\end{aligned}
$$

$$
\begin{aligned}
D= & h\left([1-\theta E(x)]^{2}-\frac{\lambda[1-2 \theta E(x)]}{P}\right. \\
& \left.-\frac{\lambda(E(x))^{2}(1-\theta)^{2}}{P_{1}}\left[1+\frac{\lambda}{P_{1}}\right]\right) \\
& +\frac{h_{1}(E[x])^{2} \lambda(1-\theta)^{2}}{P_{1}} .
\end{aligned}
$$

\section{The optimal solution $\left(n^{*}, Q^{*}\right)$ of $E[n, Q]$}

When $n$ is given, Chiu et al. [1] obtain the optimal replenishment lot size $Q^{*}(n)$ as:

$$
Q^{*}(n)=\sqrt{\frac{2\left(K+(n+1) K_{1}\right) \lambda}{A-\frac{B}{n}+D}} .
$$

So, the optimal solution $\left(n^{*}, Q^{*}\right)$ of $E[n, Q]$ can be obtained by:

$$
E\left[n^{*}, Q^{*}\right]=\underset{n \geq 1}{\operatorname{minimum}}\left\{E\left[n, Q^{*}(n)\right]\right\} .
$$

If we treat $n$ as a continuous variable, Eqs. (8) and (9) yield:

$$
\begin{aligned}
& \frac{d Q^{*}(n)}{d n} \\
& =\frac{\left(K_{1} \lambda\right) \frac{\sqrt{A-\frac{B}{n}+D}}{\sqrt{2\left(K+(n+1) K_{1}\right) \lambda}}-\frac{B \sqrt{2\left(K+(n+1) K_{1}\right) \lambda}}{\left(2 n^{2}\right) \sqrt{A-\frac{B}{n}+D}}}{A-\frac{B}{n}+D},
\end{aligned}
$$




$$
\begin{aligned}
\frac{d E\left[n, Q^{*}(n)\right]}{d n}= & \frac{K_{1} \lambda}{(1-\theta E[x]) Q^{*}(n)} \\
& -\frac{\psi_{2}(n)}{\left(Q^{*}(n)\right)^{2}} \frac{d Q^{*}(n)}{d n} \\
& +\frac{B Q^{*}(n)}{2 n^{2}(1-\theta E[x])}+\psi_{1}(n) \frac{d Q^{*}(n)}{d n}
\end{aligned}
$$

Incorporating Eqs. (10) and (11), we have:

$$
\begin{aligned}
& \frac{d E\left[n, Q^{*}(n)\right]}{d n}=\frac{K_{1} \lambda \sqrt{A-\frac{B}{n}+D}}{(1-\theta E[x]) \sqrt{2\left(K+(n+1) K_{1}\right) \lambda}} \\
& +\frac{B \sqrt{2\left(K+(n+1) K_{1}\right) \lambda}}{2 n^{2}(1-\theta E[x]) \sqrt{A-\frac{B}{n}+D}} \\
& =\frac{2 K_{1} \lambda n^{2}\left(A-\frac{B}{n}+D\right)+2 B\left(K+(n+1) K_{1}\right) \lambda}{2 n^{2}(1-\theta E[x]) \sqrt{2\left(K+(n+1) K_{1}\right) \lambda} \sqrt{A-\frac{B}{n}+D}} \\
& =\frac{2 \lambda\left[K_{1} n^{2}(A+D)+B\left(K+K_{1}\right)\right]}{2 n^{2}(1-\theta E[x]) \sqrt{2\left(K+(n+1) K_{1}\right) \lambda} \sqrt{A-\frac{B}{n}+D}} .
\end{aligned}
$$

Eq. (12) illustrates that if $B \geq 0$, then $E\left[n, Q^{*}(n)\right]$ is increasing with respect to $n \geq 1$. So, based on Eq. (9), we have:

$$
\begin{aligned}
E\left[n^{*}, Q^{*}\right] & =E\left[1, Q^{*}(1)\right] \\
& =\underset{n \geq 1}{\operatorname{minimize}}\left\{E\left[n, Q^{*}(n)\right]\right\} \text { if } B \geq 0 .
\end{aligned}
$$

Consequently, if $B \geq 0$, we have $n^{*}=1$ and $Q^{*}=$ $Q^{*}(1)=\sqrt{\frac{2\left(K+2 K_{1}\right) \lambda}{A-B+D}}$. Therefore, we conclude that the optimal number of shipments is $n^{*}=1$ if $B \geq 0$. Hence, we obtain the following results.

Theorem 1: Suppose that $B \geq 0$. Then:

(i) $E\left[n, Q^{*}(n)\right]$ is increasing with respect to $n \geq 1$;

(ii) The optimal solution, $\left(n^{*}, Q^{*}\right)$, of $E[n, Q]$ can be expressed by Eq. (13), that is $\left(n^{*}, Q^{*}\right)=$ $\left(1, Q^{*}(1)\right)=\left(1, \sqrt{\frac{2\left(K+2 K_{1}\right) \lambda}{A-B+D}}\right) ;$

(iii) The optimal number of shipments of the proposed model is $n^{*}=1$.

Conversely, if $B<0$, there exist two cases:

Case (I): If $A+D \leq 0$, Eq. (12) implies $\frac{d E\left(n, Q^{*}(n)\right)}{d n}<$ 0 for all $n \geq 1$. So, $E\left[n, Q^{*}(n)\right]$ is decreasing on $n \geq 1$. Therefore, $\left(n^{*}, Q^{*}\right)=(\infty, \infty)$;
Case (II): If $A+D>0$, Eq. (12) implies:

$$
\frac{d E\left[n, Q^{*}(n)\right]}{d n} \begin{cases}<0 & \text { if } 0<n<\Omega, \\ =0 & \text { if } n=\Omega, \\ >0 & \text { if } n>\Omega,\end{cases}
$$

where:

$$
\Omega=\sqrt{\frac{-B\left(K+K_{1}\right)}{K_{1}(A+D)}} .
$$

Eqs. (14a), (14b), and (14c) reveal that $\frac{d E\left[n, Q^{*}(n)\right]}{d n}$ is decreasing on $(0, \Omega]$ and increasing on $[\Omega, \infty)$. Let $n_{0}=$ $\lfloor\Omega\rfloor=$ the greatest integer $\leq \Omega$. Then, we have the following results.

Theorem 2: Suppose that $B<0$. Then:

Case (I): If $A+D \leq 0$, then $\left(n^{*}, Q^{*}\right)=(\infty, \infty)$;

Case (II): If $A+D>0$, then $E\left[n^{*}, Q^{*}\right]=$ $\operatorname{minimum}\left\{E\left[n_{0}, Q^{*}\left(n_{0}\right)\right], E\left[n_{0}+1, Q^{*}\left(n_{0}+1\right)\right]\right\}$. That is, $\left(n^{*}, Q^{*}\right)=\left(n_{0}, Q^{*}\left(n_{0}\right)\right)$ or $\left(n_{0}+1, Q^{*}\left(n_{0}+1\right)\right)$ associated with the less cost.

\section{Numerical examples}

In this paper, all instances in Cárdenas-Barrón et al. [2] are used for comparison. The data are shown in Table 1 . Table 2 reveals that all optimal solutions are consistent with those in Cárdenas-Barrón et al. [2].

\section{Conclusion}

Cárdenas-Barrón et al. [2] treated the long-run average costs per unit time for the model of Chiu et al. [1] as a function of the replenishment lot size, $Q$, and the number of shipments $n$. Since $n$ is restricted to being an integer number, they did not use the differential calculus approach to find the optimal solution $\left(n^{*}, Q^{*}\right)$. Basically, they adopted the approach of García-Laguna et al. [6] and used Eqs. (13) and (14) in CárdenasBarrón et al. [2] to develop the algorithm for locating the optimal solution $\left(n^{*}, Q^{*}\right)$. However, this paper considered $n$ as a continuous variable and used the differential calculus approach to obtain Theorems 1 and 2 to locate the optimal solution $\left(n^{*}, Q^{*}\right)$. There exist two cases:

Case (I): When $B \geq 0$, then $\left(n^{*}, Q^{*}\right)=\left(1, Q^{*}(1)\right)$;

Case (II): When $B<0$ and $A+D>0$, then:

$\left(n^{*}, Q^{*}\right)$

$$
=\operatorname{minimize}\left\{\left(n_{0}, Q^{*}\left(n_{0}\right)\right),\left(n_{0}+1, Q^{*}\left(n_{0}+1\right)\right)\right\},
$$


Table 1. Data of the instances in Cárdenas-Barrón et al. (2012) [2].

\begin{tabular}{ccccccc}
\hline \multirow{2}{*}{ Parameter } & $\begin{array}{c}\text { Instance 1 from } \\
\text { Chiu et al. (2012) }\end{array}$ [1] & Instance 2 & Instance 3 & Instance 4 & Instance 5 & Instance 6 \\
\hline$C$ & 100 & 190 & 21 & 100 & 120 & 300 \\
$C_{R}$ & 60 & 170 & 5 & 50 & 110 & 100 \\
$C_{s}$ & 20 & 100 & 4 & 25 & 80 & 20 \\
$C_{T}$ & 0.1 & 40 & 3 & 40 & 60 & 5 \\
$K$ & 20000 & 4000 & 400 & 65690 & 4000 & 89000 \\
$K_{1}$ & 4350 & 1 & 0.1 & 0.5 & 1 & 9.8 \\
$h$ & 20 & 30 & 10 & 10 & 90 & 150 \\
$h_{1}$ & 40 & 20 & 30 & 3 & 80 & 50 \\
$\lambda$ & 3400 & 560 & 210 & 300 & 400 & 600 \\
$P$ & 60000 & 590 & 260 & 300 & 800 & 1200 \\
$P_{1}$ & 2200 & 360 & 130 & 200 & 200 & 300 \\
$x$ & $U(0,0.3)$ & $U(0,0.21)$ & $U(0,0.11)$ & $U(0,0.4)$ & $U(0,0.69)$ & $U(0,0.35)$ \\
$E(x)$ & 0.15 & 0.105 & 0.055 & 0.2 & 0.345 & 0.175 \\
$\theta$ & 0.1 & 0.3 & 0.26 & 0.4 & 0.65 & 0.2 \\
$E(1 /(1-x))$ & 0.18889164 & 1.12248730 & 1.0593983 & 1.27706405 & 1.6973666 & 1.23080833 \\
$E(x /(1-x))$ & 0.1889164 & 0.12248730 & 0.0593983 & 0.27706405 & 0.6973666 & 0.23080833 \\
$E\left(x^{2} /(1-x)\right)$ & 0.0389164 & 0.01748730 & 0.00493983 & 0.07706405 & 0.3523666 & 0.0550833 \\
\hline
\end{tabular}

Table 2. The optimal solution of $E[n, Q]$.

\begin{tabular}{ccccccc}
\hline Parameters & \multicolumn{7}{c}{ Instance } \\
\cline { 2 - 7 } & $\mathbf{1}$ & $\mathbf{2}$ & $\mathbf{3}$ & $\mathbf{4}$ & $\mathbf{5}$ & $\mathbf{6}$ \\
\hline$B$ & 10.325275 & -1.523229 & -0.104973 & -0.92400 & -20.07692 & -0.11625 \\
$A$ & -0.29032 & 39.65082 & 5.532814 & 23.16403 & 53.42922 & 43.47971 \\
$D$ & 17.99790 & 0.98289 & 1.88041 & -0.41120 & 23.80281 & 54.25375 \\
$\Omega$ & - & 12.24683 & 7.52697 & 73.04401 & 32.25033 & 3.286856 \\
$n_{0}$ & 1 & 12 & 7 & 73 & 32 & 3 \\
$\psi_{3}$ & 374512.58883 & 141306.76304 & 5159.51912 & 47217.39130 & 101974.86304 & 198668.39378 \\
$\psi_{2}\left(n_{0}\right)$ & 99065989.84772 & 2320371.70883 & 85389.06361 & 21432717.39130 & 2079535.93297 & 55361160.62176 \\
$\psi_{2}\left(n_{0}+1\right)$ & - & 2320949.92256 & 85410.36827 & 21432880.43478 & 2080051.56300 & 55367253.88601 \\
$\psi_{1}\left(n_{0}\right)$ & 3.74736 & 21.04318 & 3.76799 & 12.37254 & 50.18333 & 50.65918 \\
$\psi_{1}\left(n_{0}+1\right)$ & - & 21.03814 & 3.76704 & 12.37245 & 50.17107 & 50.65416 \\
$Q^{*}\left(n_{0}\right)$ & 5141.61287 & 332.06497 & 150.53795 & 1316.16125 & 203.56518 & 1045.37842 \\
$Q^{*}\left(n_{0}+1\right)$ & - & 332.14613 & 150.57573 & 1316.17120 & 203.61527 & 1045.48774 \\
$E\left[n_{0}, Q^{*}\left(n_{0}\right)\right]$ & 413047.57481 & 155282.17086 & 6293.97144 & 79785.91957 & 122406.01888 & 304584.41390 \\
$E\left[n_{0}+1, Q^{*}\left(n_{0}+1\right)\right]$ & - & 155282.23779 & 6293.96978 & 79785.92110 & 122406.05676 & 304584.99488 \\
$\left(n^{*}, Q^{*}\right)$ & $\left(1, Q^{*}(1)\right)$ & $\left(12, Q^{*}(12)\right)$ & $\left(8, Q^{*}(8)\right)$ & $\left(73, Q^{*}(73)\right)$ & $\left(32, Q^{*}(32)\right)$ & $\left(3, Q^{*}(3)\right)$ \\
\hline
\end{tabular}

where:

$$
n_{0}=\left\lfloor\sqrt{\frac{-B\left(K+K_{1}\right)}{K_{1}(A+D)}}\right\rfloor .
$$

Compared with the solution procedure for Cases 1-1 and 1-2 described in Cárdenas-Barrón et al. [2], the one shown by Theorems 1 and 2 in this paper is simpler. In addition, numerical examples illustrate that our solution procedure is rather accurate. A relevant managerial insight is that we consider the number of shipments in the paper as a decision variable and also as an integer value. Future research directions are in line for applying our proposed solution procedure to other inventory models and also extending it to more realistic situations such as considering different types of 
variable demand, e.g. stock-dependent demand, creditlinked demand, and advertisement-dependent demand. These are some interesting research directions on which studies can be done in the near future.

\section{References}

1. Chiu, W.C., Chiu, Y.-S.P. and Yang, J.C. "Combining an alternative multi-delivery policy into economic production lot size problem with partial rework", Expert Systems with Applications, 39(3), pp. 25782583 (2012).

2. Cárdenas-Barrón, L.E., Taleizadeh, A.A. and TreviñoGarza, G. "An improved solution to replenishment lot size problem with discontinuous issuing policy and rework, and the multi-delivery policy into economic production lot size problem with partial rework", Expert Systems with Applications, 39(18), pp. 1354013546 (2012).

3. Sana, S.S. "A collaborating inventory model in supply chain", Economic Modelling, 29(5), pp. 2016-2023 (2012).

4. Sana, S.S. "An economic order quantity model for nonconforming quality products", Service Science, 4(4), pp. 331-348 (2012).

5. Sana, S.S. and Goyal, S.K. " $(Q, r, L)$ model for stochastic demand with lead-time dependent partial backlogging", Annals of Operations Research, 233(1), pp. 401-410 (2015).

6. García-Laguna, J., San-José, L.A., Cárdenas-Barrón, L. E. and Sicilia, J. "The integrality of the lot size in the basic EOQ and EPQ models: Applications to other production-inventory models", Applied Mathematics and Computation, 216(5), pp. 1660-1672 (2010).

\section{Biographies}

Kun-Jen Chung is a Full Professor of the College of Business at Chung Yuan Christian University. He was awarded a $\mathrm{PhD}$ in Industrial Management from Georgia Institute of Technology. His interests include Markov decision processes, economic designs of control charts, inventory control, and reliability. He has had over 172 articles accepted/published in national and international journals.

Pin-Shou Ting is currently a Professor at Department of International Business Management in Shih Chien University. He has published several papers and technical notes in national and international journals.

Leopoldo Eduardo Cárdenas-Barrón is currently a Professor at School of Engineering and Sciences at Tecnológico de Monterrey, Campus Monterrey, México. $\mathrm{He}$ is also a Faculty Member in the Department of Industrial and Systems Engineering at Tecnológico de Monterrey. He was the Associate Director of the Industrial and Systems Engineering programs from 1999 to 2005. Moreover, he was also the Associate Director of the Department of Industrial and Systems Engineering from 2005 to 2009 . His research areas include inventory planning and control primarily, logistics, and supply chain. He has published several papers and technical notes in national and international Journals. He has co-authored one book in the field of Simulation in Spanish. He is also an Editorial Board Member in several international journals. 